\title{
Tomotherapy (Slice Therapy)
}

\author{
Maha Esmeal Ahmed ${ }^{1}$, Mwahib Sid Ahmed Mohammed Osman Aldosh ${ }^{2}$ \\ 1 Ph. D, Assistant Professor-Radiological Sciences Department- Applied Medical College, Najran University- \\ Kingdom of Saudi Arabia \\ 2 Ph. D, Assistant Professor,Co-ordinator of Radiological Sciences Department Applied Medical College - \\ Najran University- Kingdom of Saudi Arabia
}

\begin{abstract}
: homotheraphy "slice therapy" is the proposal for the delivery of radiation therapy with intensitymodulated strips of radiation. The proposed method employs alinear accelerator, which would be mounted on aring gantry like a helical tomotherapy (CT scanner). The patient would move through the bore of the gantry simultaneously with gantry rotation. The intensity modulation would be performed by temporally modulated multiple independent leaves that open and close across the slit opening. This method would result in the delivery of highly conformal radiation and overall treatment times should be comparable with contemporary treatment delivery times. The dose reconstruction capabilities provide an ability to determine the dose actually delivered to the patient. The ring gantry would make it convenient to mount anarrow multisegmental megavoltage detector system for beam verification and aCTscanner on the treatment unit. A treatment unit could become a powerful tool for treatment planning, conformal treatment and verification using tomographic images. In this review a full description of the development of this novel technique is given, properties, advantages and applicability of this device are evaluated and the fundamental design specifications are justified. There is no previous studies for that purpose in Saudi Arabia so, researchers recommend that king Khalid hospital in Najran to implement Tomotheraphy unit in the near future for improving imaging and treatment planning .

Key words: Tom therapy units advantages planning conformal verification treatment.
\end{abstract}

\section{Introduction:}

The term tomotherapy refers to both the technique and the trade name of the device .

Thomotheraphy is a new system delivery therapy with ahelical diagnostic CT scan ,in which the patient and radiation source move simultaneously. The intensity of the radiation beam is modulated by a computer controlled mechanism.

It provides unprecedented accuracy in the beam delivery allowing for an increase in tumor dose there by increasing the likelihood of cancer cure while at the same time reducing treatment complications in healthy tissues. It provides also significant advantages over today's state-of-the-art radiation treatment, in addition to on-line imaging which allows for treatment adaptation on a daily basis accounting for the tissue locations on each set-up. In this review, advances in radiation therapy for cancer are reviewed because it is important and there is no previous studies in this field in Saudi Arabia particularly in Najran region .The paper clarify the definition of Tomotherapy, history of its development ,advantages, future potential of technological innovations in radiotherapy and unique features of helical tomotherapy from patient perspective are reviewed. And the past, present and future of tomotherapy are discussed. The purposes of this review is to explore the applications of improved imaging tools in treatment planning using tomotherapy technology and to highlight future challenges and directions in the clinical use of radiotherapy.

\section{Tomotherapy units:}

The tomotherapy unit fits into a significantly smaller room compared to modern linear accelerators since it does not involve a couch rotation. Because of the CT detectors with an added beam stopper and the ring mounting the primary beam is virtually fully attenuated thereby reducing the shielding requirements of the treatment. Both the reduced room size and the reduction of shielding will provide significant cost savings in the implementation of this technology. Because tomotherapy is a single energy linac fully integrated with a treatment planning system it is expected that once the technology becomes routine it will be significantly easier to commission in comparison to today's multi-energy and multi-modality (photons and electrons) linacs.

It uses a two step process which incorporates image guided targeting of the tumor together with radiation treatment in one seamless progression. The two step process usually takes 15 minutes and is as follows:

1. Imageguided Positioning: A diagnostic quality CT image is acquired on the Tomotherapy unit immediately before each treatment. This localizes a patient's anatomy (identifies the tumor size and shape) with the precision of a CT scan, resulting in a high accuracy of pretreatment positioning and tumor targeting. If the tumor's size or shape changes during treatment, radiotherapy parameters can be modified to adapt to this change. 


\section{TomoTherapy:}

After the targeting procedure has verified the target location by examining the anatomy of a patient's body, the Tomotherapy System uses a helical 360 degree rotating beam of radiation where by the intensity of the radiation treatment beam is continuously modulated or controlled to conform to the shape of the tumor while minimizing radiation.

\section{Treatment planning:}

Cancer is the most significant health care problem in the western world surpassing heart disease as the leading cause of potential years of life lost [1]. In Canada, about 134,000 people are diagnosed annually with cancer. This represents more than one in three people who will develop cancer during their lifetimes. Radiation will be used to treat approximately 66,000 new cancer patients per year of whom 33,000 will be treated with an attempt to cure the disease. Before beginning a Tomotherapy treatment, the doctor puts together a plan using 3D images from a combination of scanning technologies (such as CT and MRI) and special software to establish the precise contours for each tumor and surrounding sensitive organs or tissues. The doctor then prescribes how much radiation the tumor should receive, as well as acceptable levels for healthy tissue nearby. The Tomotherapy treatment system calculates the appropriate pattern, position and intensity of the radiation beams to be delivered. Based on the doctors prescribed dose. The treatment system is an all-in-one solution, incorporating software for treatment planning, quality assurance, patient set-up and treatment delivery. It facilitates the first fully-integrated process for radiation therapy, reducing the number of technical decisions that need to be made and giving clinicians more time to focus on patients. Tomotherapy provides slice-by-slice treatment through the marriage of two technologies: spiral CT scanning and Intensity Modulated Radiation Therapy. Operating much like a CT scanner by rotating around the patient, the device provides radiation, produced and delivered by a linear accelerator in a spiral delivery pattern. The division of beams creates dose distributions at more angles and with better precision than ever before. The integration of imaging, treatment planning, patient positioning and treatment in one system can reduce the burden to patients by shortening the course of treatment and curtailing side effects $[2,3]$.

Helical TomoTherapy is an advanced radiation oncology technology that can ease treatment regimens for patients, reducing costs and in some cases, yield better cancer control than traditional methods of radiation which focus on tumors from a limited number of angles and with wider beams of radiation, necessitating the delivery of small, divided doses of radiation over a long period of time in order to spare adjacent healthy tissue. Tomotheraphy process is complex and involves multiple steps. The process begin with patient diagnosis and three dimensional (3-D)imaging through various steps that prepare the patient for treatment and finally to treatment verification and actual radiation dose delivery. The first implementation of this concept was performed by NOMOS Corporation [4,5].

Intensity-modulated radiotherapy (IMRT) [6) has been introduced [4-7 ]into a wide spectrum of clinics worldwide. It is clear that recently its adoption has accelerated due to the availability of commercial planning and delivery software from all the major treatment planning and Linac manufacturers. A full description of the development and milestones of this novel technique is given by Webb [8] and more recently in the first article in this series [9].

\section{Conformal treatment:}

TomoTherapy can be used to treat tumors of various sizes, single or multiple tumors, one region of the body or several regions, to the same dosage in every area or to multiple different radiation dosages. TomoTherapy is currently being used to treat select tumors in the prostate, brain, head and neck, lung, liver, pancreas, prostate, uterus as well as other sites. One of the most exciting aspects of the TomoTherapy system is that tumors can be treated radiosurgically. The patient slowly moves through the center of the gantry, elerator comes around, the beam is directed at a slightly different plane. Radiation dose is modulated at every angle to conform to the target. The popular introduction of IMRT started in the mid 1990s when the NOMOS Corporation (Swickley, Pennsylvania, USA) introduced the PEACOCK system [10, 11],.Static beam delivery, arcs and tomotherapy There are a handful of studies in the literature that seek to systematically compare delivery of conformal radiotherapy by static beam and arc based (such as tomotherapy) techniques. Verellen et al [12] conclude that for convex target geometries that whereas both modalities achieve treatment goals, dynamic (conformal) arcs fare better than static beam IMRT delivery with respect to treatment efficiency. As their name suggests the latter utilize arc based solutions, furthermore at each "arc position" the MLC leaf positions are adjusted to provide appropriate conformal (target) shielding. For more complicated (concave) treatment geometries the efficiency advantage was maintained though, as expect. In both cases serial tomotherapy plans provided good solutions though the treatment efficiency was compromised because of the high number of monitor units required. Pirzkall [13] noted that rotational IMRT proved to have a slight advantage over fixed field IMRT. Philosophically, the interesting aspect might draw from these statements is 
that the use of arc based therapy is useful in providing highly conformal treatments and the addition of intensity modulation simply adds to this basic advantage. Of course, this concept has been previously investigated and indeed used clinically, though on a very small scale. Intensity-modulated arc therapy (IMAT) was proposed by $\mathrm{Yu}[14,15]$. Here, in addition to the conformal shaping of the MLCs during the arc, multiple arc passes are delivered in order to lay down more than a single intensity level. Arguably, widespread clinical adoption has been slowed by the fact that the treatment planning manufacturers have not yet provided solutions for this methodology. MacKenzie and Robinson [16] added an interesting facet to this discussion, they describe a method for intensity modulated arc deliveries approximated by a large number of fixed gantry position sliding window dynamic multileaf collimator fields. The premise that should want to deliver Tomotherapy-like distributions using conventional equipment is itself interesting.

There is no previous studies for that purpose in Saudi Arabia so, researchers recommend that king Khalid hospital to implement Tomotheraphy unit in Najran area in the near future for improving imaging and treatment planning and without these modality doctors cannot check the size and shape and location of the tumors before each treatment.

\section{Verification treatment:}

Adaptive radiotherapy is a novel application that enables TomoTherapy to target high-dose radiation in cases where sparing healthy tissue is critical. Tumors change in size and anatomical formation throughout the course of radiation treatment. TomoTherapy differs from other tissue-sparing techniques with daily CT imaging used to plot the tumor's exact contours. This allows the oncologists to assess the tumor's size and shape in real time and make any necessary adjustments to further tighten the treatment field and conform to the tumor, resulting in greater protection to healthy tissue. This strategy is in contrast to the traditional practice of enlarging the treatment field to defend against missing any part of the tumor. TomoTherapy can expose normal tissues to less radiation than traditional radiation methods[1].

The indications for treatment with Tomotherapy include :Lung and Breast Cancers, Head \& Neck Cancers, Gynecologic Cancers, Sarcomas, Rectal Cancers, prostate cancer, Multiple Metastases (more than one lesion) and Retreatment of previous maximally-treated tumors. The imaging advantages, that Tomotherapy treatment system lets clinicians efficiently acquire 3D CT images of every patient, every day. With these images, clinicians can check the size, shape and location of tumors before each treatment. Then they can compare that day's image with the one used for planning, to make sure that radiation will be directed to where it should be. Importantly, daily CT images can also be used to analyze and, if necessary, modify a patient's treatment at any point during the treatment course. Benefits for the Patient: Verification of patient position prior to each therapy session via onboard CT scanner, large field size, radiation therapy with the precision and versatility of a CT scanner minimized radiation exposure to healthy tissue. Benefits for the Physicians: Pretreatment image verification (Tomo Image): gives the physician the confidence of knowing the size and shape of a patient's lesion each treatment day, combines a highly advanced treatment planning, system with advanced conformal treatment delivery .Potential disadvantages of tomotherapy is not be appropriate for all patients requiring radiotherapy. In IMRT, though the region that is exposed to high dose radiation is highly conformal, low doses of radiation are typically spread over a larger volume of normal tissue compared with conventional radiation treatments. Therefore, the total absorbed dose to the individual, called the integral dose, is much higher in patients receiving. IMRT. As IMRT is a new technology, the absolute risks of a higher integral dose are not completely known at this time. In general, most tissues tolerate low doses of radiation well and the risk of acute or chronic damage to exposed normal tissues is very small. However, following exposure to low dose radiation there is a slightly increased risk of radiation-induced second malignancies years or even decades following treatment. Though this risk is very small for adults, it is significantly higher in pediatric and young adult patients. Therefore, when the potential benefits of a more precise treatment plan are small, a conventional radiation plan with a lower integral dose may be preferable [17].

\section{Cancer and radiation therapy:}

The London Regional Cancer Centre in (27 October 2001) celebrated the fiftieth anniversary of the first patient in the world treated with cobalt-60. The London Regional Cancer Centre was pleased to be part of the tomotherapy developments in radiation therapy. It is also exciting to note that two Canadian centres, Edmonton and London, will be the first in the world to install helical tomotherapy units, other than in Madison where the system is being developed.

\section{Advances in head and neck cancer radiotherapy}

TomoTherapy can be used to treat a variety of cancers and for tumors of any size, from malignancies in confined areas, such as the prostate, to larger regions with multiple metastases. The technology is particularly well-suited to treating the head, neck, lungs and pelvis. Radiation for cancers of the tongue, throat and larynx is 
associated with damage to salivary glands and side effects such as dry mouth and pain. TomoTherapy's ability to provide image-guided sculpting of radiation in three dimensions within a complex anatomy prevents damage to adjacent tissues, which has been difficult to do in these cases using other radiation treatments [1].

TomoTherapy also be advantageous in treating cervical and endometrial cancers in order to protect the bowel, bladder and rectum and reduce the risk of troublesome side effects. For lung cancer, the technology minimizes the risks of breathing problems and damage to the esophagus. While TomoTherapy is most often associated with reducing side effects and shortening treatment time, it may also improve cancer control in some types of malignancies. Lung cancer outcomes may be enhanced with TomoTherapy because it allows for increasing daily radiation doses. TomoTherapy can also be used to repeat radiation treatment to areas of the body that have been previously irradiated. This approach reduces the risk of scarring, ulceration and pain associated with repeat irradiation[1] (figure 1).

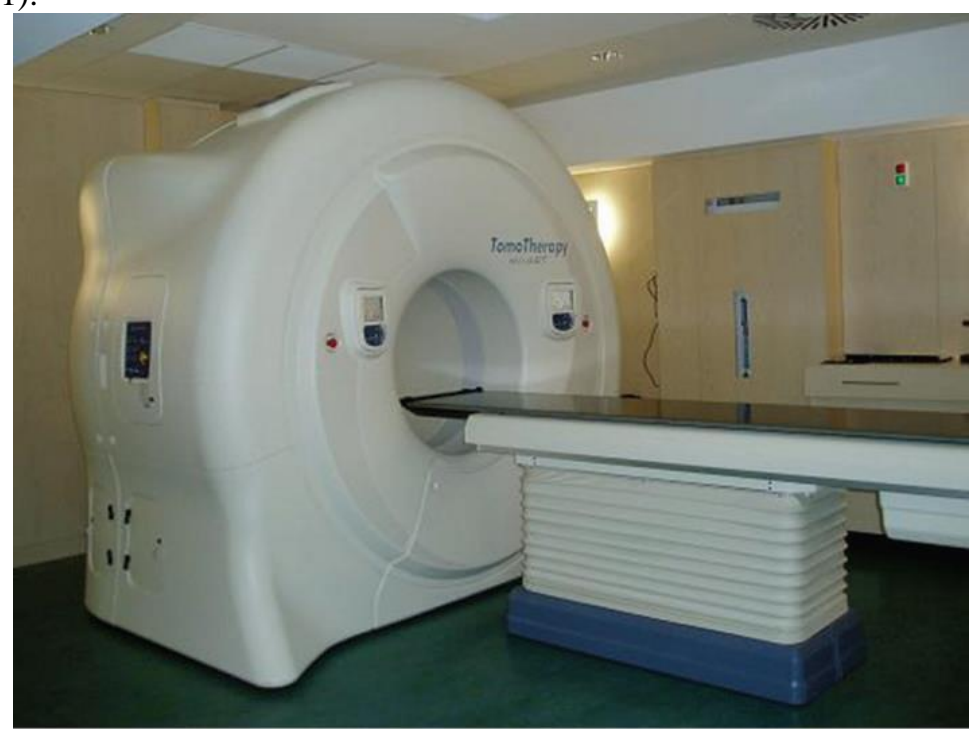

Figure 1: TomoTherapy Treatment Systems

\section{External beam radiation therapy:}

During the past 50 years, the most common form of radiotherapy has been external beam radiation therapy (EBRT). Conventional EBRT uses a beam of high energy $\mathrm{x}$-rays or photons directed at the tumor from one to several different directions, so that radiation is focused on a target and surrounding healthy tissue is shielded. EBRT is typically delivered via a linear accelerator, a machine which produces and focuses photons towards the target, with photons exerting their effects by ionization of molecules in tissues directly within their path (Figure 2).

Conventional radiation therapy machines deliver a wide beam of radiation from only a few angles. The treatment system uses its unique CT scanner design to

deliver radiation continuously from all angles around the patient. It features patented beam-modulating technology that divides a single beam into many smaller,

narrow beamlets. More angles and more precise modulation result in dose distributions that conform to tumors like never before. This, in turn, minimizes damage to surrounding healthy tissue. Recently, TomoTherapy Inc. developed a novel approach to IMRT. While conventional radiation therapy machines have been adapted and readapted to keep up with advances in the fi eld, the new TomoTherapy unit was

designed without legacy constraints. Tomotherapy system is a new, advanced, state of the art, helical IMRT delivery system with CT image guidance. The system was developed at the University of Wisconsin-Madison and the first patient was treated in 2003. (Figure 2) 


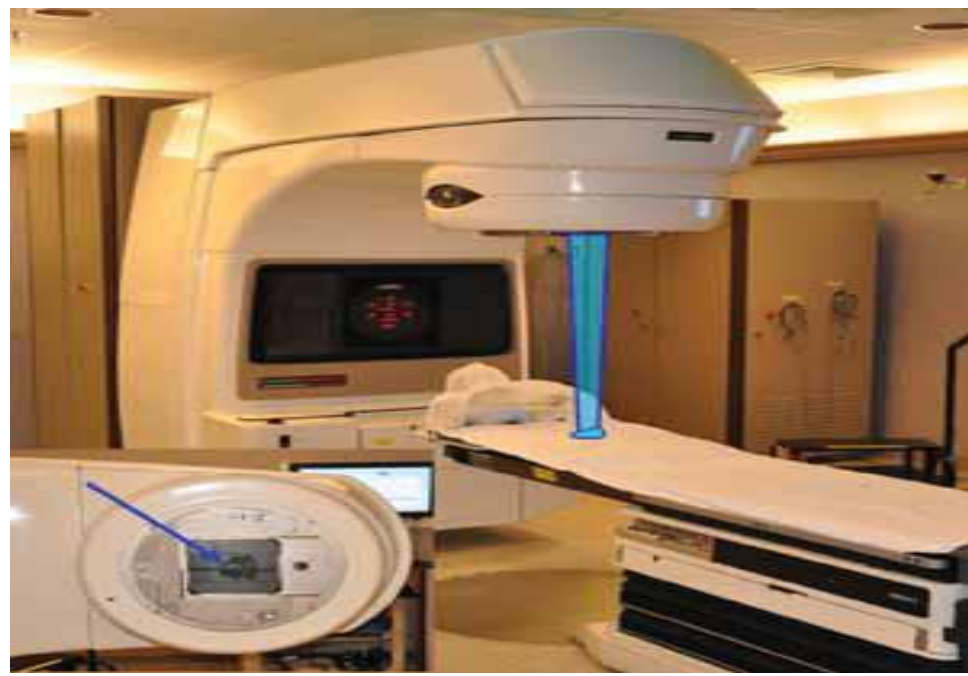

Figure 2. A 6-18MV conventional linear accelerator

\section{Tomo therapy in Saudi Arabia:}

There is no any previous studies in that purpose in saudia Arabia researches found some information about. The installation of tomo therapy's, a next-generation radiation therapy solution aimed at improving the precision of cancer care, at King Faisal Specialist Hospital \& Research Center (KFSH\&RC) in Riyadh, Saudi Arabia. According to Tom therapy, Saudi Arabia is the first in the Middle East region to treat patients with the Tomo therapy system. Also ,the Department of Radiation Therapy Oncology Center at the King Fahd Medical City is currently working on the introduction of modern technologies in the field of radiation therapy , helical IMRT delivery system with CT image guidance. The Department of Radiation Therapy Oncology Center of the largest radiotherapy departments in Saudi Arabia [18].

There is no previous studies for that purpose in Saudi Arabia so researchers did not find any information concern tomotherapy so they recommend that king Khalid hospital to implement Tomotheraphy unit in Najran area in the near future for improving imaging and treatment planning and without these modality doctors cannot check the size and shape and location of the tumors before each treatment and because radiation oncology is evolving rapid development over the world.

\section{Conclusion:}

The technology of radiation oncology is evolving at a rapid rate, primarily as a result of the evolution of computer applications and their integration into diagnostic imaging and radiation therapy dose delivery equipment. Perhaps the Tomotherapy development represents the greatest advance in radiation therapy since the first use of cobalt-60 in the 1950s. These advances will provide a radiation treatment technology that allows daily adaptation of the treatment technique to match the location of the tumor and the normal tissues with the better ability to focus the radiation beams, higher doses can be delivered to the tumor resulting in higher cure rates. In addition, lower doses will be delivered to normal tissues resulting in lower complication rates. The net result should be an overall improvement in the quality of life of the cancer patient With this advanced technology can sculpt powerful and precise radiation beams to treat hard-to-reach tumors. Using built-in CT scanning to confirm the shape and position of the tumor before each treatment, Tomotherapy reduces radiation exposure to healthy tissues and organs and many cancer patients who have reached their maximum tolerance dose of traditional radiation may be a candidate for Tomotherapy radiation. Lastly researches recommend that king Khalid hospital in the Najran area to implement Tomotheraphy unit in the near future for improving imaging and treatment planning and without these modality doctors cannot check the size and shape and location of the tumors before each treatment and because radiation oncology is evolving rapid development over the world.

\section{Acknowledgement :}

Having completed this research, would like to acknowledge all those who directly or indirectly extend support to the research. Thanks for prof .Kang Jin Oh-South Korea,Seoul-Kyunghee university Special thanks to Prof.Mohamed Alfateh Esmeal Ahmed for his support and great help. Researchers would like also to extend our gratitude to the Dean ship and staff of the College of Medical Radiological Sciences, Najran University. The researchers gratefully acknowledges family, husbands and all those who contribute to this work but their names are not mentioned, and we won them special gratitude. May almighty Allah make this 
research of special benefit to the development of health services all over the world.

\section{References :}

[1]. W. Grant III, R.B. Cain. Intensity Modulated Conformal Therapy for Intracranial Lesions. Med. Dosim. 23, 237-241, 1998.

[2]. National Cancer Institute, Canadian Cancer Statistics 2001, Toronto, Canada, 2001. Also located on the internet at http://www.cancer.ca/

[3]. M.P. Carol, Peacock: A System for Planning and Rotational Delivery of Intensity-Modulated Fields. Int. J. Imag. Sys. Technol. 6, 56-61 (1995). See alsohttp://www.nomos.com/

[4]. Intensity Modulated Radiation Therapy Collaborative Working Group. Intensity-modulated Radiotherapy: current status and issues of interest. Int J Radiat Oncol Biol Phys 2001;51:880-914.

[5]. James HV, Scrase CD, Poynter AJ. Practical experience with intensity-modulated radiotherapy. Br J Radiol 2004;77:3-14.

[6]. Beavis A. IMRT - in our real (NHS) world? RAD magazine. 2003 September:33-34

[7]. McNair HA, Adams EJ, Clark CH, Miles EA, Nutting CM. Implementation of IMRT in the radiotherapy department. Br J Radiol 2003;76:850-6.

[8]. Webb S. Intensity modulated radiotherapy. Bristol: Institute of Physics Publishing, 2000.

[9]. Webb S. The physical basis of IMRT and inverse planning. Br J Radiol 2003;76:678-89.

[10]. Carol M. Peacock: a system for planning and rotational delivery of intensity- modulated fields. Int J Imag Syst Technol 1995;6:56-61.

[11]. Woo SY, Butler B, Grant III W. Clinical experience: benign tumours of the CNS and head and neck tumours. In: Sternick ES, editor. Intensity modulated radiation therapy. Madison, WI: Advanced Medical Publishing; 1997:195-8

[12]. Verellen D, Linthout N, Soete G, Van Acker S, De Roover P, Storme G. Considerations on treatment efficiency of different conformal radiation therapy techniques for prostate cancer. Radiother Oncol 2002;63:27-36.

[13]. Pirzkall A, Carol M, Lohr F, Hoss H, Wannenmacher M, Debus J. Comparison of intensity-modulated radiotherapy with conventional conformal radiotherapy for complexshaped tumors. Int J Radiat Oncol Biol Phys 2000;48: 1371-80.

[14]. Yu C. Intensity-modulated arc therapy with dynamic multileaf collimation: an alternative to tomotherapy. Phys Med Biol $1995 ; 40: 1435-49$

[15]. Yu C, Allen L. Clinical implementation of intensity modulated arc therapy. Int J Radiat Oncol Biol Phys 2002; 53:453-63.

[16]. MacKenzie MA, Robinson DM. Intensity modulated arc deliveries approximated by a large number of fixed gantry position sliding window dynamic multileaf collimator fields. Med Phys 2002;29:2359-65.

[17]. 17.www.thefreelibrary.com

[18]. 18.http://www.businesswire.com/news/home/20090324006402/en/TomoTherapy-Announces-Middle-East-Installation$\mathrm{Hi} \% \mathrm{C} 2 \% \mathrm{~B} 7 \mathrm{Art} \% \mathrm{C} 2 \% \mathrm{AE}-\mathrm{Cancer}$ 